\title{
ABNORMALITIES OF ONE-CARBON METABOLISM IN DEPRESSION: A FAMILY CLINICAL CASE
}

\author{
Evgeny D. Kasyanov ${ }^{1}$, Tatyana V. Zhilyaeva ${ }^{2}$ \& Galina E. Mazo ${ }^{1}$ \\ ${ }^{I}$ V.M. Bekhterev National Medical Research Center for Psychiatry and Neurology, \\ Saint Petersburg, Russia Federation \\ ${ }^{2}$ Privolzhskiy Research $\square$ Medical $\square$ University, Nizhny $\square$ Novgorod, $\square$ Russian Federation \\ received: 7.4.2021; \\ revised: 17.7.2021; \\ accepted: 25.7.12.2021
}

\section{INTRODUCTION}

Abnormalities of one-carbon metabolism (OCM) are some of the well-studied metabolic factors in the pathophysiology of depression (Bhatia \& Singh 2015). OCM consists of several interconnected biochemical cycles, the main task of which is to transfer methyl groups from one molecule to another. Folate deficiency and elevated homocysteine levels play an essential role in development of OCM abnormalities (Ducker GS \& Rabinowitz 2017). Currently a sufficiently large amount of evidence has been received showing that abnormalities of OCM of various origins (folate and vitamin B12 deficiency in food, genetic polymorphisms of folate cycle enzymes, including MTHFR677TT) can be a risk factor for affective disorders, in particular depression.

Hyperhomocysteinemia (HHcy) is the most reliable serum marker of OCM abnormalities. A large number of studies have shown a relationship between high plasma homocysteine and depression (Chen et al. 2005), including several population studies (Miller 2008; Kim et al. 2008). The association of low folate levels with high homocysteine levels with the risk of depression has also been confirmed in a number of population studies (Sachdev et al. 2005, Kim et al. 2008). Moreover, a number of studies have shown that among patients with endogenous depression, the frequency of occurrence of the MTHFR gene defect and HHcy is significantly higher than in the general population (Chen et al. 2005, Lewis et al. 2006).

\section{CASE REPORT}

A 23-year-old white man with a family history of mood disorders suffered from treatment-resistant depression for two years. He received consistently the trials of several selective serotonin reuptake inhibitors - SSRIs (fluoxetine, paroxetine, fluvoxamine, sertralin, citalopram and escitalopram) and serotonin-norepinephrine reuptake inhibitors - SNRIs (venlafaxine, milnacipran, duloxetine), tricyclic antidepressant (amitriptyline), Monoamine oxidase inhibitors - MAOIs (pirlindole), and also pregabalin and quetiapine. The therapeutic effect was always incomplete: the patient still suffered from anhedonia and decreased sexual interest.

By the time of the consultation, the patient had not been taking therapy. As a result of assessment with Mini International Neuropsychiatric Interview (MINI) he was diagnosed with: recurrent depressive disorder, social anxiety disorder and general anxiety disorder. The Montgomery-Asberg Depression Rating Scale (MADRS) score was 39 points (severe depression). Considering the positive response to venlafaxine, we chose to use this drug with increasing the dose to the maximal tolerable and to evaluate further effect.

The mother of the patient - a 53-year-old white woman had similar symptoms of depression. The recent decline 1,5 years ago. By the time of consultation she had not taken any therapy. As a result of assessment with MINI she was diagnosed with: recurrent depressive disorder and general anxiety disorder. MADRS score - 33 points (moderate depression).

During the clinical interview, it was also revealed that earlier, when a neurologist prescribed injections of $B$ vitamins, the patient noticed a significant improvement in her condition. That is why it was suggested that the patient and her son should conduct an examination to determine the levels of vitamins B9, B12 and homocysteine in the blood. The mother and son were found to have HHcy (see Table 1), and the son also had a decreased concentration of plasma folates. After genetic analysis, heterozygous variants of the gene of methylenetetrahydrofolate reductase MTHFR (C677T and A1298C) and heterozygous variant of the gene of methionine reductase MTRR (A66G) were found.

Considering the findings, mother and son were prescribed adjuvant therapy with methylfolate at a dose of 400 and $800 \mathrm{mg}$, respectively. Also, escitalopram at a dose of $10 \mathrm{mg}$ was prescribed to the mother of the patient because of its antidepressant and antianxiety effects.

At the time of the prescription of methylfolate, the son was taking venlafaxine at a dose of $225 \mathrm{mg}$ /day for 
Table 1. Blood and genetic test results for mother and son with depression

\begin{tabular}{lccccccc}
\hline No & $\begin{array}{c}\text { Homocysteine } \\
\mu \mathrm{mol} / \mathrm{l}(\text { reference values } \\
4.44-13.56 \mu \mathrm{mol} / \mathrm{L})\end{array}$ & $\begin{array}{c}\text { Vit B9 nmol/l } \\
(\text { reference values } \\
7-39.7 \mathrm{nmol} / \mathrm{l})\end{array}$ & $\begin{array}{c}\text { Vit B12 pg/ml } \\
(\text { reference values } \\
187-883 \mathrm{pg} / \mathrm{ml})\end{array}$ & $\begin{array}{c}\text { MTHFR } \\
(\mathrm{C} 677 \mathrm{~T})\end{array}$ & $\begin{array}{c}\text { MTHFR } \\
(\mathrm{A} 1298 \mathrm{C})\end{array}$ & $\begin{array}{c}\text { MTRR } \\
(\mathrm{A} 66 \mathrm{G})\end{array}$ & $\begin{array}{c}\text { MTR } \\
(\mathrm{A} 2756 \mathrm{G})\end{array}$ \\
\hline $\begin{array}{l}\text { No 1 } \\
\text { mother }\end{array}$ & $15.09 \uparrow$ & 11.74 & 250 & $C / T$ & $A / C$ & $A / G$ & $\mathrm{~A} / \mathrm{A}$ \\
$\begin{array}{l}\text { No 2 } \\
\text { son }\end{array}$ & $40.72 \uparrow$ & $4.34 \downarrow$ & 242 & $C / T$ & $A / C$ & $A / G$ & $\mathrm{~A} / \mathrm{A}$ \\
\hline
\end{tabular}

3 weeks - without a significant clinical improvement. By the $7^{\text {th }}$ week of treatment (4 weeks of taking methylfolate), the patient had a significant improvement (MADRS - 14 points), including a significant decrease in the severity of anhedonia. The homocysteine level was $9.07 \mu \mathrm{mol} / \mathrm{L}$ after 3 months from the start of therapy with methylfolate; the patient was in remission. In the case of the mother, by the $4^{\text {th }}$ week of treatment with escitalopram and methylfolate, there was a decrease of MADRS scores to 4 . No signs of affect inversion and hypomania were found. After 1.5 months from the beginning of therapy the homocysteine level was 7.53 $\mu \mathrm{mol} / \mathrm{L}$; the patient was in remission.

\section{DISCUSSION}

The two clinical cases illustrate family forms of OCM abnormalities and depression. We can pinpoint no striking distinctive features in the course of depression in these patients with comorbid OCM abnormalities. Depression in the patients had a typical melancholic structure with anhedonia and somatic symptoms (decreased appetite and sleep disturbances, such as early awakening). All depressive symptoms decreased significantly in the course of a combined therapy with antidepressant and methylfolate, after which the patients had a remission for the first time in a long time.

Adjuvant therapy used in these patients deserves a separate discussion. The choice of the form of folate as the drug of choice is discussed in the scientific literature, and to date there are no recommendations based on evidence-based studies. Methylfolate (5MTHF, 5-methyltetrahydrofolate, metapholine) is a biologically active form of folates, the closest to the folates of natural food, which easily penetrates the central nervous system (unlike synthetic folic acid and food dihydrofolate), does not require preliminary transformation induced by the dihydrofolate reductase enzyme in the liver, it is immediately incorporated into the folate exchange cycle and, in addition, its further biochemical exchange (in particular, the supply of methyl groups to the homocysteine-methionine cycle) is independent of the MTHFR enzyme, therefore, it allows to avoid the problems of transformation in carriers of genetic polymorphism MTHFR677C $>$ T (Nelson 2012). Therefore, in a large number of studies on the effect of folate on mood, methylfolate has been in the focus.
One of the limitations of this work is the lack of blinding, which does not make it possible to exclude a placebo effect in patients and distortions in the doctor's assessment of the condition of patients who take adjuvant therapy with methylfolate.

\section{CONCLUSION}

Mood disorders are often associated with OCM abnormalities. Patients with treatment-resistant depression need additional laboratory tests (folic acid, cyanocobalamin and homocysteine) to rule out OCM abnormalities. Further long-term, high-quality, randomized clinical trials of folates (folic acid, methyl folate, etc.) and SAM with double blinding and placebo control in patients with depression are still needed.

\section{Acknowledgements: None.}

\section{Conflict of interest: None to declare.}

\section{Contribution of individual authors:}

Evgeny D. Kasyanov was involved with the patient's care, conception and design, data collection, manuscript preparation and writing the paper.

Tatyana V. Zhilyaeva was involved with data collection, reviewed draft manuscript and manuscript preparation.

Galina E. Mazo was involved with conception and design, manuscript preparation and reviewed draft manuscript.

\section{References}

1. Bhatia P, Singh N: Homocysteine excess: delineating the possible mechanism of neurotoxicity and depression. Fundam Clin Pharmacol 2015; 29:522-8

2. Chen CS, Tsai JC, Tsang HY, Kuo YT, Lin HF, Chiang IC, Devanand DP: Homocysteine levels, MTHFR C677T genotype, and MRI Hyperintensities in late-onset major depressive disorder. Am J Geriatr Psychiatry 2005; 13:869-75

3. Ducker GS, Rabinowitz JD: One-Carbon Metabolism in Health and Disease. Cell Metab. 2017 Jan 10;25:27-42

4. Kim JM, Stewart R, Kim SW, Yang SJ, Shin IS, Yoon JS: Predictive value of folate, vitamin $B 12$ and homocysteine levels in late-life depression. Br J Psychiatry 2008; 192:268-74 
5. Lewis SJ, Lawlor DA, Davey Smith G, Araya R, Timpson $N$, Day IN, Ebrahim $S$ : The thermolabile variant of MTHFR is associated with depression in the British Women's Heart and Health Study and a meta-analysis. Mol Psychiatry 2006;11:352-60.

6. Miller AL: The methylation, neurotransmitter, and antioxidant connections between folate and depression. Altern Med Rev 2008; 13:216-26
7. Nelson JC: The evolving story of folate in depression and the therapeutic potential of l-methylfolate. Am J Psychiatry 2012; 169:1223-5

8. Sachdev PS, Parslow RA, Lux O, Salonikas C, Wen W, Naidoo D, Christensen H, Jorm AF: Relationship of homocysteine, folic acid and vitamin B12 with depression in a middle-aged community sample. Psychol Med 2005;35:529-38

\section{Correspondence:}

Evgeny D. Kasyanov, MD

V.M. Bekhterev National Medical Research Center for Psychiatry and Neurology 192019, Saint Petersburg, 3 Bekhterev St., Russian Federation

E-mail: ohkasyan@yandex.ru 